\title{
Impact of the COVID-19 pandemic on gastrointestinal endoscopy in the Netherlands: analysis of a prospective endoscopy database
}

Authors

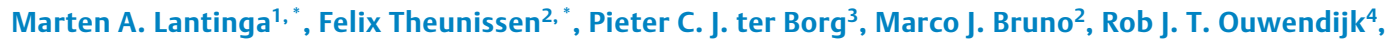
Peter D. Siersema ${ }^{1}$, on behalf of the Trans.IT foundation study group ${ }^{\#}$

Institutions

1 Department of Gastroenterology and Hepatology, Radboud University Medical Center, Nijmegen, The Netherlands

2 Department of Gastroenterology and Hepatology, Erasmus Medical Center, Rotterdam, The Netherlands

3 Department of Gastroenterology and Hepatology, Ikazia Ziekenhuis, Rotterdam, The Netherlands

4 Department of Gastroenterology and Hepatology, Bravis Ziekenhuis, Roosendaal, The Netherlands

submitted 2.7.2020

accepted after revision 8.9 .2020

published online 20.10 .2020

Bibliography

Endoscopy 2021; 53: 166-170

DOI 10.1055/a-1272-3788

ISSN 0013-726X

(C) 2020. Thieme. All rights reserved.

Georg Thieme Verlag KG, Rüdigerstraße 14,

70469 Stuttgart, Germany

Scan this QR-Code for the author commentary.

Tables 1s, 2s

Supplementary material is available under

https://doi.org/10.1055/a-1272-3788
Corresponding author

Peter D. Siersema, MD, PhD, Department of Gastroenterology and Hepatology, Radboud University Medical Center, P.O. Box 9101, 6500 HB Nijmegen, The Netherlands

Fax: +31-24-3540103

Peter.Siersema@radboudumc.nl

\section{ABSTRACT}

Background COVID-19 has dramatically affected gastrointestinal endoscopy practice. We aimed to investigate its impact on procedure types, indications, and findings.

Methods We retrospectively analyzed endoscopies performed in 15 Dutch hospitals by comparing periods 15 March to 25 June of 2019 and 2020 using the prospective Trans.IT database.

Results During lockdown in 2020, 9776 patients underwent endoscopy compared with 19296 in 2019. Gastroscopies decreased by $57 \%$ (from 7846 to 4467 ) and colonoscopies by $45 \%$ (from 12219 to 5609), whereas endoscopic retrograde cholangiopancreatography volumes remained comparable (from 578 to 522). Although endoscopy results indicative of cancer decreased (from 524 to 340), the likelihood of detecting cancer during endoscopy increased (2.7\% [95\% confidence interval $(\mathrm{Cl}) 2.5-3.0$ ] in 2019 versus $3.5 \%[95 \% \mathrm{Cl} 3.1-3.9]$ in $2020 ; P<0.001)$. After lifting of lockdown, endoscopy volumes started to return to normal, except for colorectal cancer screening.

Conclusions Fewer endoscopies were performed during the COVID-19 lockdown, leading to a significant reduction in the absolute detection of cancer. Endoscopies increased rapidly after lockdown, except for colorectal cancer screening.

\footnotetext{
* These authors contributed equally to this work.

\# The Trans.IT foundation study group: M. Kerkhof (Groene Hart Ziekenhuis, Gouda); R.M.E. Slangen (HagaZiekenhuis, Den Haag), P.J. Bus (Laurentius Ziekenhuis, Roermond); F.J.G.M. Kubben (Maasstad Ziekenhuis, Rotterdam); W.J. Thijs (Martini Ziekenhuis, Groningen); M.J.M. Groenen (Rijnstate Ziekenhuis, Arnhem), E. A. Hoboken (Rode Kruis Ziekenhuis, Beverwijk), W. Bruins Slot (Spaarne Gasthuis, Haarlem), L. Wormmeester (Treant Zorggroep, Hoogeveen), F. Vleggaar (UMC Utrecht, Utrecht), L.A. Noach (Ziekenhuis Amstelland, Amstelveen), J.J. Uil (Ziekenhuis Gelderse Vallei, Ede); M. Rasica (Bravis Ziekenhuis, Roosendaal); M.E. van Leerdam (Antoni van Leeuwenhoek, Amsterdam and Leiden University Medical Center, Leiden); E.J. Kuipers (Erasmus Medical Center, Rotterdam).
} 


\section{Introduction}

SARS-CoV-2 RNA virus has resulted in the largest pandemic in modern history [1]. COVID-19 has so far been confirmed in nearly 32 million individuals, with over 971000 deaths worldwide by the end of September 2020 [2]. As a consequence, COVID-19 continues to have a dramatic effect on healthcare utilization. The almost exclusive care of infected patients requiring hospitalization and mechanical ventilation has considerably changed the routine way of working in healthcare facilities of affected countries, including endoscopy units [3,4].

In the Netherlands, the first confirmed case was reported on 27 February 2020, which was followed by an exponential increase in COVID-19 patients in the following weeks. On 12 March, the Dutch Government proclaimed a nationwide lockdown. This prompted a large initiative to prepare all healthcare facilities for the upcoming inflow of COVID-19 patients and to minimize activities not related to COVID-19 by reducing outpatient clinics, including a suspension of the nationwide colorectal cancer (CRC) screening program on 16 March [5]. As a consequence, by the end of March, a dramatic reduction in endoscopic procedures was observed as all elective procedures were cancelled or postponed.

Our aim was to investigate the impact of the COVID-19 lockdown period on the type, indication, and findings of endoscopic procedures and to determine the effect on gastrointestinal (GI) cancer detection. We also investigated which endoscopic procedures and findings increased as soon as the lockdown was (partially) lifted on 11 May 2020.

\section{Methods}

\section{Database and data collection}

We analyzed data from the Trans.IT database (Rotterdam, The Netherlands). This anonymized multicenter database was set up in 2012 and currently collects GI endoscopy data from 20 Dutch hospitals (three academic and 17 nonacademic hospitals). All participating sites use a uniform structured reporting tool developed by Trans.IT and incorporated into the reporting system of Endobase (Olympus Europe, Hamburg, Germany) to report endoscopy findings. The structured reporting tool was further improved by Trans.IT to provide extended ICD-10 codes for all diagnoses and interventions, allowing for systematic and uniform data collection [6]. Following each endoscopy, endoscopists are required to create a report on the endoscopic findings [7,8]. All anonymized endoscopy reports are automatically uploaded to the database [9]. Patient characteristics, endoscopy details, and findings are subsequently extracted from individual endoscopy reports and automatically stored in the database. Currently, the Trans.IT database annually collects more than 150000 endoscopy reports on average, including approximately 63000 gastroscopies, 64000 colonoscopies (of which 14500 are CRC screening colonoscopies), and 5300 endoscopic retrograde cholangiopancreatography (ERCP) procedures.

Data from 2019 and 2020 of 15 of the 20 participating hospitals (including two academic hospitals) were available for data analysis for this study. None of the registered endoscopic procedures were excluded from analysis.

\section{Outcomes and definitions}

The main outcome for this study was the number of procedures performed. Other outcomes were the indications and findings of the endoscopic procedures.

To investigate the effect of the Dutch lockdown, we compared the periods 15 March-15 May in both 2019 and 2020. To evaluate the effect of the partial lifting of lockdown measures, we additionally investigated the periods 15 May-25 June 2019 and 2020. Of particular interest were the findings of endoscopically suspected esophageal, gastric, colon, and rectal cancer, Barrett's esophagus, peptic ulcer disease, and colorectal polyps (one polyp or more). Histology reports were not available for analyses. Furthermore, we also extracted indications for colonoscopies, which were divided into four main categories: hematochezia, surveillance (including history of polyp removal, CRC, or inflammatory bowel disease), familial/inherited syndromes, and nonspecific. We also identified the number of colonoscopies performed following a positive fecal immunochemical test as part of the Dutch CRC screening program.

\section{Statistics}

SPSS statistical software package version 25 (IBM Corp., Armonk, New York, USA) was used for statistical analyses. Data were imported from the internal search module of the Trans.IT database, which is hosted by a collaboration of Radventure (Utrecht, The Netherlands) and Bitam (Tampica, Mexico) and accessed through the "KPI online" tool provided by Bitam [10].

Categorical variables were reported as frequencies (\%) with $95 \%$ confidence intervals ( $\mathrm{Cls}$ ), and nonparametric data were reported as median with interquartile range. Data analyses were performed by categorizing the sample into the years 2019 and 2020. We used the chi-squared test for categorical data and Mann-Whitney $U$ test for nonparametric data. A twosided $P$ value of $<0.05$ was considered statistically significant.

\section{Ethical considerations}

The collection of patient data in the Trans.IT database was approved by the privacy officer of the Erasmus Medical Center and follows the Dutch Personal Data Protection Act. All data are anonymously stored in a secure environment and not traceable to a person without disproportionate effort. Formal ethical review was therefore waived.

\section{Results}

\section{Endoscopy characteristics}

We had access to the endoscopy database of 15 Dutch hospital, which in 2019 performed a total of 120571 endoscopies in 94 586 patients. Median age of patients and the percentage of male and female patients were generally comparable in both periods 15 March-15 May of 2019 and 2020 (see Table 1s in the online-only supplementary material). For the period 15 March - 15 May, a large decrease in the number of patients undergoing endoscopy was seen in 2020 compared with 2019 
(from 19296 to 9776). Gastroscopy and colonoscopy volumes decreased to $57 \%$ (from 7846 to 4467 ) and $45 \%$ (from 12219 to 5609), respectively, whereas the number of ERCPs remained relatively unchanged (from 578 in 2019 to 522 in 2020).

\section{Endoscopy results}

- Fig. 1 illustrates the decrease in the absolute number of endoscopy findings with a diagnosis "suspicious of $\mathrm{Gl}$ cancer" when comparing $2019(n=524)$ and $2020(n=340)$. A steep decline in the absolute number of suspected colon cancer cases was seen (from 299 in 2019 to 168 in 2020). However, in patients who did undergo endoscopy in 2020, a suspicion of $\mathrm{Gl}$ cancer was diagnosed significantly more often compared with 2019 (3.5\% [95\%Cl 3.1-3.9] vs. 2.7\% [95\%Cl 2.5-3.0]; $P<$ 0.001 ) (Table $1 \mathrm{~s})$. Specifically, a relative increase in a suspicion of gastric and rectal cancer was observed in 2020 vs. 2019 (gastric cancer $0.4 \%$ [ $95 \% \mathrm{Cl} 0.3-0.6]$ vs. $0.3 \%$ [ $95 \% \mathrm{Cl} 0.2-0.3]$, respectively, $P=0.01$; and rectal cancer $0.6 \%[95 \% \mathrm{Cl} 0.4-0.7]$ vs. $0.3 \%[95 \% \mathrm{Cl} 0.2-0.4]$, respectively, $P<0.001$ ), whereas the number of suspected gastric and rectal cancer cases remained almost equal (42 vs. 50 and 55 vs. 56, respectively).

During the lockdown period, colorectal polyps were less frequently observed during colonoscopy (5301 in 2019 vs. 2127 in 2020). A reduction of $19 \%$ was seen in the number of suspected peptic ulcer disease cases, although its frequency in relation to the number of endoscopies performed significantly increased from $0.8 \%(95 \% \mathrm{Cl} 0.7-0.9$ ] in 2019 to $1.3 \%$ [ $95 \% \mathrm{Cl} 1.1-1.5$ ] in 2020; $P<0.001)$.

Although colon surveillance was the main indication for colonoscopy in $2019(35.0 \%, 95 \% \mathrm{Cl} 33.9-36.1)$, this indication accounted for only $19.4 \%(95 \% \mathrm{Cl} 18.1-20.8$; $P<0.001)$ of colonoscopies in 2020. During the 2020 lockdown period, hematochezia became one of the more predominant indications for colonoscopy $(38.4 \%, 95 \% \mathrm{Cl} 36.7-40.1)$ compared with $26.7 \%$ (95\%Cl 25.7-27.8; $P<0.001)$ in 2019 (Table 2s).

\section{Trends in endoscopy volume}

- Fig. 2 shows the endoscopy volumes from 1 January to 25 June 2020. A steep decline was seen in the absolute number of endoscopies performed in the Netherlands during the COVID19 lockdown (period between the dashed vertical lines on - Fig. 2). In particular, a massive drop in gastroscopy and colonoscopy volumes was seen in April 2020. After the partial lifting of lockdown on 11 May, gastroscopy and colonoscopy volumes started to return to pre-pandemic levels. In contrast, the volume of CRC screening colonoscopies remained low on 25 June. ERCP volume remained unchanged throughout this period ( 1 January to 25 June 2020).

\section{Discussion}

This retrospective analysis including 15 Dutch endoscopy units illustrates the direct effect of the COVID-19 pandemic lockdown on endoscopy volumes, with a subsequent reduction in the number of Gl cancers detected. Nonetheless, if patients underwent endoscopy during the lockdown period, they were more likely to be diagnosed with a suspected cancerous lesion.

We observed a significant absolute reduction in endoscopic procedures during the lockdown period from March to May 2020, particularly gastroscopies and colonoscopies. This is at least partially explained by postponed or cancelled elective endoscopic procedures at the time when infections rapidly spread throughout the Netherlands. This finding corresponds with observations from other countries [11]. Another important factor was the observed decrease in patient referrals from general practitioners to the Gl outpatient clinic, resulting in a marked decline in endoscopic procedures (unpublished data). In contrast to gastroscopy and colonoscopy, the volume of ERCPs remained relatively unchanged, which might be explained by the fact that most ERCP indications are emergencies, and delays are not therefore advisable for these patients.

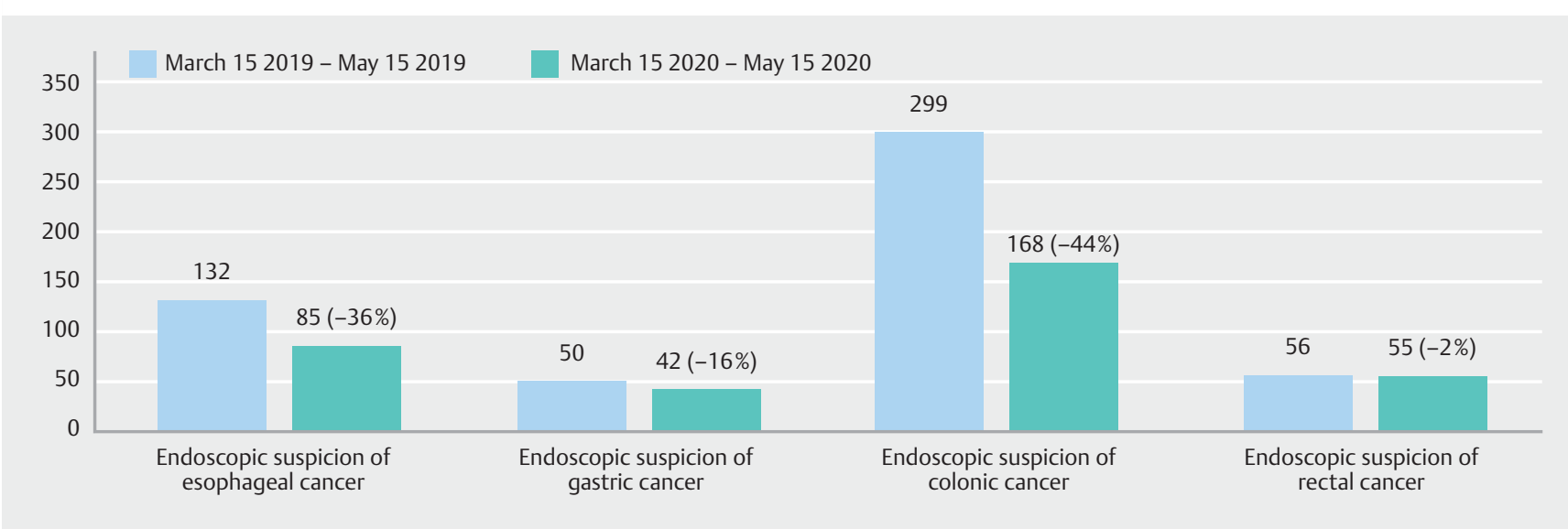

- Fig. 1 Absolute number of endoscopy results showing a suspicion of gastrointestinal cancer when comparing the period 15 March - 15 May of 2019 and 2020, stratified by type of cancer. 

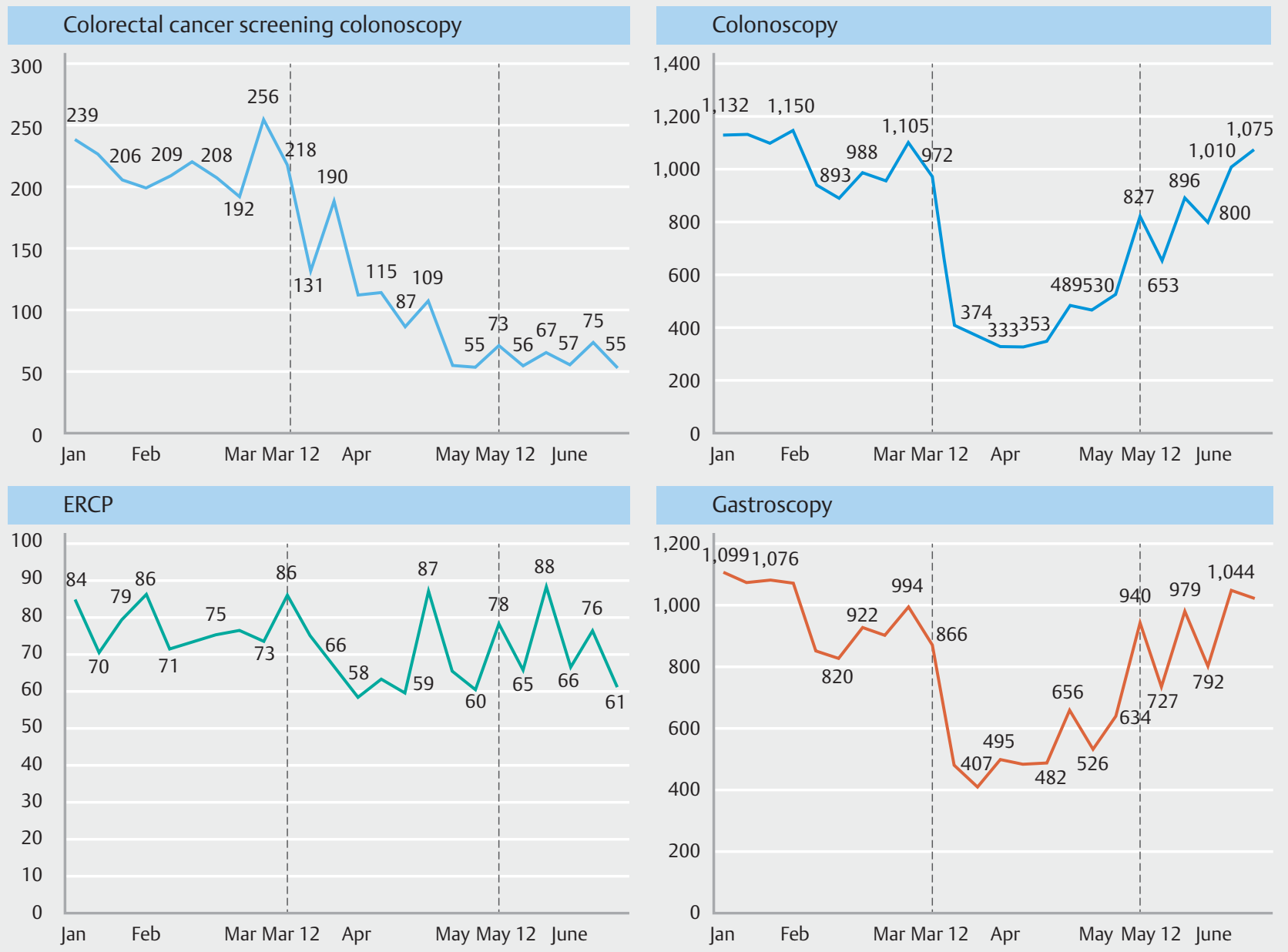

- Fig. 2 Volume of different gastrointestinal endoscopy procedures during the COVID-19 pandemic in 2020 (1 January-25 June). Dashed lines correspond to initiation and partial release of Dutch national lockdown. ERCP, endoscopic retrograde cholangiopancreatography.

The Dutch CRC screening program was first introduced in 2014 and has run continuously ever since [12]. However, the nationwide lockdown resulted in a temporary pause of the screening program on 16 March. A dramatic decrease in the number of endoscopically suspected colon cancer cases was subsequently seen. From the beginning of June, the Dutch government decided to gradually restart the program; however, even by the end of June, no increase in screening colonoscopies was observed. The true impact of the relatively short interruption of the screening program remains uncertain, although even a modest delay in diagnosis and treatment of CRC has been shown to have a negative impact on long-term survival [13].

A worrisome observation was the evident decrease in absolute number of patients with an endoscopically suspected diagnosis of Gl cancer. Delay of cancer diagnosis will negatively affect initial staging, therapeutic options, and ultimately patient survival. However, the percentage of patients undergoing endoscopy and in whom a cancer diagnosis was suspected increased significantly compared with 2019. This suggests that endoscopic procedures in the Netherlands were still being performed for patients presenting with symptoms consistent with malignancy. Moreover, we observed a relatively unchanged absolute number of diseases that are often associated with GI bleeding, for example peptic ulcer disease, gastric cancer, and rectal cancer. This is in contrast, however, with findings from Austria, where a $40 \%$ decline in upper Gl bleeds was seen during the lockdown [14]. Nonetheless, our results show that despite a decrease in overall endoscopic procedures, this probably did not result in a significant change in the clinical management of acute life-threatening situations such as a peptic ulcer bleeding or symptomatic gastric and rectal cancer; however, long-term follow-up results are needed to confirm this.

Following (partial) lifting of the lockdown, a marked increase in endoscopic procedures was observed. However, despite the presence of waiting lists due to postponed procedures, this did not lead to overuse of endoscopy services in the first 6 weeks after the (partial) easing. As was also observed in a survey among North American endoscopists [15], a persistent lack of protective personal equipment and other resources may have prevented a further increase in endoscopy volumes to compensate for the backlog of elective procedures. In addition, it is possible that nonmalignant indications for endoscopy 
might no longer be considered a sufficient reason to perform an endoscopy. Finally, we and other participating centers have observed that patients are still reluctant to undergo endoscopy and more often than before ask to postpone the procedure or cancel the appointment at short notice (unpublished results).

This study comes with some limitations. First, this was a retrospective analysis of data not primarily collected for research purposes. However, Trans.IT offers a unique prospective systematic documentation of endoscopic procedures, volumes, and outcomes $[6,7]$. Second, diagnoses were based on an endoscopic diagnosis and histopathological confirmation was not available, which could have influenced the real incidence of $\mathrm{Gl}$ cancers in this cohort. Finally, some diagnoses could have been missed; however, reporting of an endoscopic procedure without using the structured reporting tool is unlikely to have occurred because of legal obligations.

In conclusion, the COVID-19 pandemic has had a large impact on endoscopy practices in the Netherlands, and the lockdown resulted in a significant decrease in GI cancer diagnoses, particularly colon cancer. Nonetheless, in those patients who did undergo endoscopy, an increased likelihood of a GI cancer diagnosis was seen.

\section{Competing interests}

Prof. Bruno is a consultant for and has received support for industryand investigator-initiated studies from Boston Scientific and Cook Medical. He has also received support for investigator-initiated studies from Pentax Medical, Mylan, and ChiRoStim. Dr. Ouwendijk has received research grants from Janssen Netherlands, Olympus, and the Coolsingel Foundation. Prof. Siersema has received research grants from Norgine, Pentax, Microtech, Yakult, and Motus GI (ongoing), and is an advisory board member for Motus Gl. All other authors declare that they have no conflicts of interest.

References

[1] Zhou P, Yang X-L, Wang X-G et al. A pneumonia outbreak associated with a new coronavirus of probable bat origin. Nature 2020; 579: 270-273

[2] Medicine JHUo Coronavirus Resource Center. COVID-19 dashboard by the Center for Systems Science and Engineering (CSSE) at Johns Hopkins University (JHU). Baltimore: Johns Hopkins University of Medicine; https://coronavirus.jhu.edu/map.html Last accessed September 2020

[3] Gralnek IM, Hassan C, Beilenhoff U et al. ESGE and ESGENA Position Statement on gastrointestinal endoscopy and the COVID-19 pandemic. Endoscopy 2020; 52: 483-490
[4] Zhang $Y$, Zhang $X$, Liu L et al. Suggestions for infection prevention and control in digestive endoscopy during current 2019-nCoV pneumonia outbreak in Wuhan, Hubei province, China. Endoscopy 2020; 52: 312-314

[5] National Institute for Health and Environment. Ministry of Health, Welfare and Sport. COVID-19 (novel coronavirus).https://www.rivm. nl/ Last accessed September 2020

[6] Groenen M], Hirs W, Becker $\mathrm{H}$ et al. Gastrointestinal endoscopic terminology coding (GET-C): a WHO-approved extension of the ICD-10. Dig Dis Sci 2007; 52: 1004-1008

[7] Groenen M], Kuipers E], van Berge Henegouwen GP et al. Computerisation of endoscopy reports using standard reports and text blocks. Neth J Med 2006; 64: 78-83

[8] Soekhoe JK, Groenen M], van Ginneken AM et al. Computerized endoscopic reporting is no more time-consuming than reporting with conventional methods. Eur J Intern Med 2007; 18: 321-325

[9] Groenen M], van Buuren HR, van Berge Henegouwen GP et al. Validation study of automatically generated codes in colonoscopy using the endoscopic report system Endobase. Scand J Gastroenterol 2010; 45: $1121-1126$

[10] Bitam KPI Online. 2020: https://kpionline.bitam.com

[11] Forbes N, Smith ZL, Spitzer RL et al. Changes in gastroenterology and endoscopy practices in response to the coronavirus 2019 pandemic: results from a North American survey. Gastroenterology 2020; 159: 772-774

[12] Schreuders EH, Ruco A, Rabeneck L et al. Colorectal cancer screening: a global overview of existing programmes. Gut 2015; 64: 1637-1649

[13] Sud A, Jones M, Broggio J et al. Collateral damage: the impact on outcomes from cancer surgery of the COVID-19 pandemic. Ann Oncol 2020; 31: 1065-1074

[14] Schmiderer A, Schwaighofer $H$, Niederreiter $L$ et al. Decline in acute upper gastrointestinal bleeding during Covid-19 pandemic after lockdown in Austria. Endoscopy 2020: doi:10.1055/a-1178-4656

[15] Kushnir VM, Berzin TM, Elmunzer B] et al. Plans to reactivate gastroenterology practices following the COVID-19 pandemic: a survey of North American centers. Clin Gastroenterol Hepatol 2020; 18: 22872294

\section{CORRECTION}

Impact of the COVID-19 pandemic on gastrointestinal endoscopy in the Netherlands: analysis of a prospective endoscopy database

Lantinga MA, Theunissen F, ter Borg PC] et al. Endoscopy 2021, 53: 166-170.

In the above-mentioned article, one sentence on page 168 has been corrected.

Correct is: Gastroscopy and colonoscopy volumes decreased to $57 \%$ (from 7846 to 4467 ) and $45 \%$ (from 12219 to 5609), respectively, whereas the number of ERCPs remained relatively unchanged (from 578 in 2019 to 522 in 2020). This was corrected in the online version on November 17, 2021. 